\title{
Exploiting Di-Muon Production at PANDA
}

\section{Destefanis ${ }^{* \dagger}$}

Università degli Studi di Torino and INFN

E-mail: marco.destefanis@to.infn.it

The physics program of the future PANDA experiment includes the investigation of the nonperturbative region of the QCD by means of antiproton beams, eventually polarised, with a beam momentum up to $15 \mathrm{GeV} / \mathrm{c}$. Part of the PANDA spectrometer is devoted to the muon identification, that allows to access many among those processes needed to probe the nucleonic structure. The high foreseen luminosity should allow to investigate the Drell-Yan (DY) production of muon pairs. This reaction is a unique tool to access the spin depending properties of the nucleon, and in particular its transverse degrees of freedom, by means of experimental asymmetries leading to the Transverse Momentum Dependent Parton Distribution Functions (TMD PDF's). Moreover, a scan across the J/psi mass region should allow a measurement of the phase between the strong and the electromagnetic amplitudes of the J/psi decay. The investigations on the azimuthal asymmetries and on the $\mathrm{J} / \mathrm{psi}$ scan expected in the PANDA scenario will be discussed in details.

8th International Conference on Nuclear Physics at Storage Rings-Stori11,

October 9-14, 2011

Laboratori Nazionali di Frascati dell'INFN, Italy

* Speaker.

${ }^{\dagger}$ For the Torino PANDA-MU Collaboration. 


\section{Experimental Scenario}

The future FAIR facility, which will be built at Darmstadt, Germany, will provide different nuclear beams. It will also be equipped with different storage rings. In one of them, the High Energy Storage Ring (HESR), antiprotons will be stored. The PANDA Collaboration $[1,2]$ is planning to take advantage of the physics potential which will be available utilizing high intensity, phase space cooled antiproton beams. The Collaboration is planning to exploit those beams, which will be provided by the new FAIR facility in a momentum range of $1.5-15 \mathrm{GeV} / \mathrm{c}$, in order to address fundamental questions on QCD as well as on electromagnetic processes.

The PANDA detector can be divided into two spectrometers: the Target Spectrometer (TS) and the Forward Spectrometer (FS). The TS surrounds the interaction region. It is designed to cover the angles between $5^{\circ}\left(10^{\circ}\right)$ in the vertical (horizontal) plane and $140^{\circ}$ in the laboratory frame and its detectors are mostly contained inside the superconducting solenoid. The muon counters (MUO) is placed in between the magnet return joke. The FS covers the most forward region in order to detect those particles emitted at small laboratory angles. Both TS and FS are provided with specific detectors for tracking the particles trajectories, detect their energy loss, and perform the particle identification.

In the design of the spectrometer, the PANDA Collaboration devoted a particular attention to the muon detection. In fact, several planes of MUO are placed in both the spectrometer regions. The muon counters are composed of Mini Drift Tubes (MDT) and of fiberglass strip boards (STRIP) [3]. The MDT are an evolution of the Iarocci tubes [1,4] in which the proportional operation mode is preferred to the streamer one, and they are provided with a metallic cathode, to avoid aging effects. The MDT volumes are filled with a $\mathrm{Ar}+\mathrm{CO}_{2}$ mixture. The STRIP is composed of doublesided copper laminated fiberglass, so to allow the read out of the second coordinate by collecting the induced charge. In order to install the strip boards, the MDT metallic cathode is removed. The layout of a MDT module is shown in Fig. 1. A full scale prototype $\left(3912 \times 2116 \times 32 \mathrm{~mm}^{3}\right)$ of
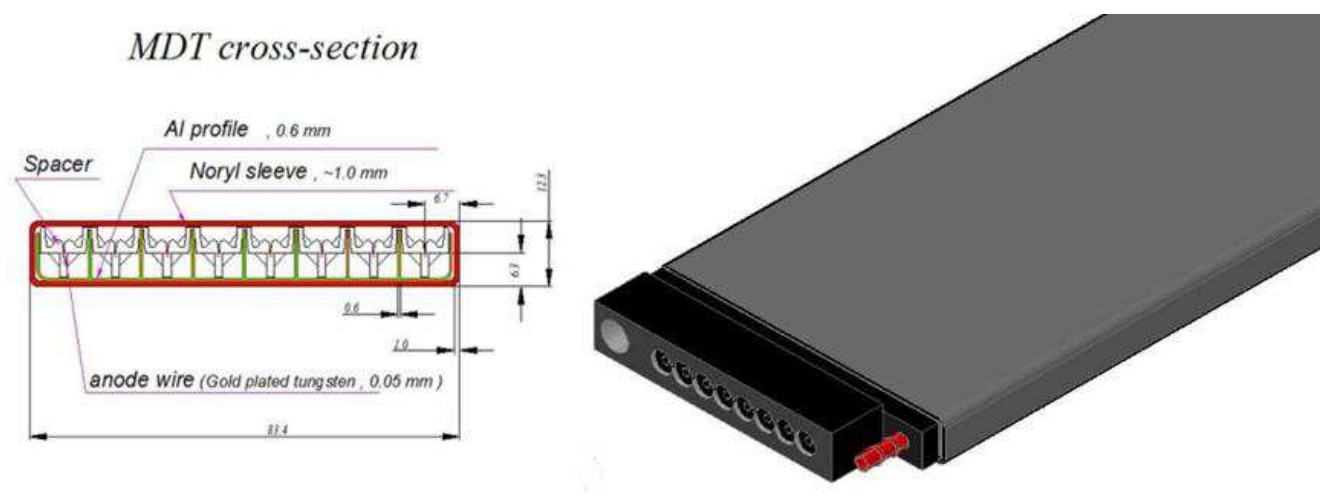

Figure 1: MDT cross section (left) and MDT module layout (right).

the muon counters has already been constructed and then transported to CERN for test purposes. To clearly understand the PANDA layout, a "Range System" prototype (RS) has been prepared as well. The RS, as shown in Fig. 2, is a segmented iron block with the dimensions $1530 \times 1060$ 


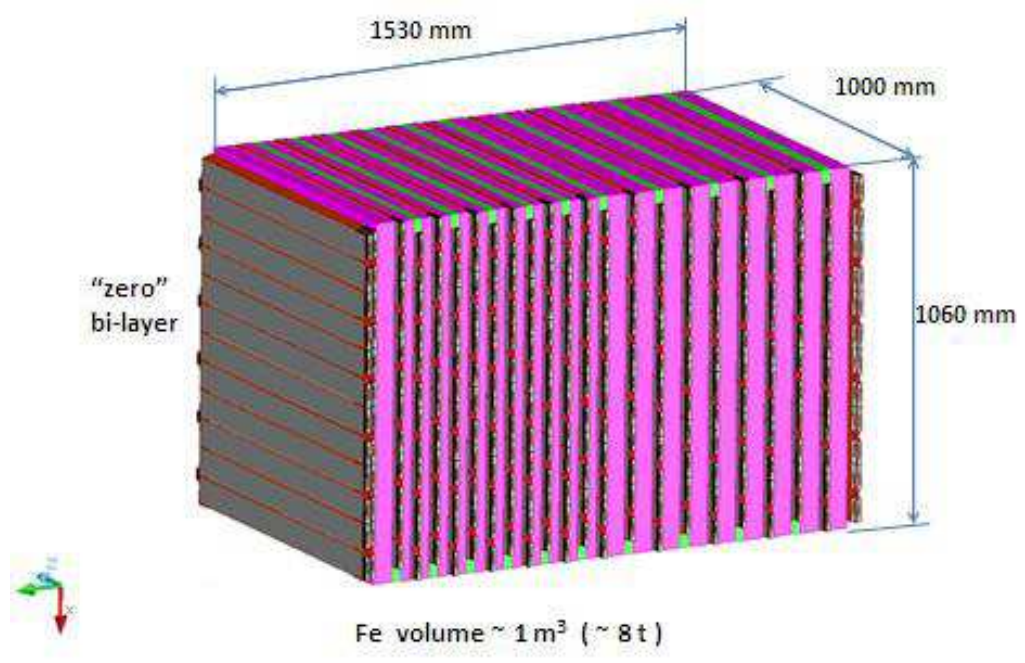

Figure 2: Range system prototype used to study the MUO setup of the PANDA Spectrometer.

$\times 1000 \mathrm{~mm}^{3}$ which reproduces the exact PANDA layout with the MUO planes in between the segmented iron volumes.

The fact that the PANDA detector is equipped with a muon detection system gives us the possibility to investigate QCD and electromagnetic processes. Two studies which will be performed with the help of muons will be here presented: the Drell-Yan and the measurement of the phase between the strong and the electromagnetic $J / \psi$ decay amplitudes.

\section{Drell-Yan Processes}

The Parton Distribution Functions (PDF) and the Fragmentation Functions (FF) are used to obtain a complete description of the nucleonic structure. At the leading twist, the quark structure of hadrons is described by three PDF's: the unpolarized function $f_{1}(x)$, the helicity $g_{1}(x)$, and the transverse polarization $h_{1}(x) . f_{1}(x)$ is the probability of finding a quark with a fraction $x$ of the longitudinal momentum of the parent hadron; $g_{1}(x)$ describes the longitudinal polarization distribution; the Transversity $h_{1}(x)$ describes the quarks' transverse spin distribution inside a transversely polarized hadron.

The hadron structure interpretation becomes more difficult when the experimental polarized cross sections [5] is investigated. Other factors, like the intrinsic transverse momentum of the partons $\left(\mathbf{k}_{T}\right)$, have to be taken into account. In a leading twist Transverse Momentum Dependence (TMD) approach, eight independent PDF's are defined as function of $x$ and $\mathbf{k}_{T}$. The TMD PDF's can be investigated in different experimental scenarios and with different beam-target configurations. In Semi-Inclusive Deep Inelastic Scattering (SIDIS) experiments, the TMD PDF's are convoluted with the FF, and that leads to experimental and theoretical challenges to their extraction. 
On the other hand, in the Drell-Yan (DY) production of lepton pairs one can define experimental asymmetries depending on the TMP PDF's only, in order to extract their distributions.

DY is an electromagnetic process in which a quark and an antiquark annihilate through a virtual photon to a lepton pair final state: $h_{1} h_{2} \rightarrow l^{+} l^{-} X$. In order to have a better identification of the DY signals, the $\mu^{+} \mu^{-} X$ channels are preferred. The DY is usually studied in the rest frame of the virtual photon, the so called Collins-Soper frame [6]. One can, then, define a hadron and a lepton plane, the initial and the final state planes respectively. Taking into account the angles between those two planes, and between the lepton plane and the nucleon spin $\left(S_{1,2}\right)$, one can define the angles $\varphi$ and $\varphi_{S_{1,2}}$, respectively.

In the completely unpolarized DY events, the differential cross section can be expressed as [2]:

$$
\begin{aligned}
\frac{d \sigma^{0}}{d \Omega d x_{1} d x_{2} d q_{T}}= & \frac{\alpha^{2}}{12 Q^{2}} \sum_{f} e_{f}^{2}\left\{\left(1+\cos ^{2} \vartheta\right) F\left[\bar{f}_{1}^{f} f_{1}^{f}\right]+\right. \\
& \left.+f \sin ^{2} \vartheta \cos 2 \varphi F\left[\left(2 \hat{\mathbf{h}} \cdot \mathbf{p}_{1_{T}} \hat{\mathbf{h}} \cdot \mathbf{p}_{2_{T}}-\mathbf{p}_{1_{T}} \cdot \mathbf{p}_{2_{T}}\right) \frac{\bar{h}_{1}^{\perp f} h_{1}^{\perp f}}{M_{1} M_{2}}\right]\right\}
\end{aligned}
$$

where $\alpha$ is the fine structure constant, $e_{f}$ is the charge of a parton with flavour $f$, and $q_{T}$ is the transverse momentum of the lepton pair. The Boer-Mulders (BM) function $h_{1}^{\perp}$ describes the distribution of transversely polarized quarks inside an unpolarized hadron, and it can be connected to the orbital motion of the parton inside the hadron. This term shows a $\cos 2 \varphi$ azimuthal dependence which contributes to the leading twist description of the cross section.

If one of the annihilating hadrons is transversely polarized, the single-polarized DY cross section can be defined as:

$$
\frac{d \sigma}{d \Omega d x_{1} d x_{2} d q_{T}}=\frac{d \sigma^{0}}{d \Omega d x_{1} d x_{2} d q_{T}}+\frac{d \Delta \sigma^{\uparrow}}{d \Omega d x_{1} d x_{2} d q_{T}},
$$

where

$$
\begin{aligned}
& \frac{d \Delta \sigma^{\uparrow}}{d \Omega d x_{1} d x_{2} d q_{T}}=\frac{\alpha^{2}}{12 s Q^{2}} \sum_{f} e_{f}^{2}\left|\mathbf{S}_{2_{T}}\right|\left\{\left(1+\cos ^{2} \vartheta\right) \sin \left(\varphi-\varphi_{S_{2}}\right) F\left[\hat{\mathbf{h}} \cdot \mathbf{p}_{2_{T}} \frac{\bar{f}_{1}^{f} f_{1 T}^{\perp f}}{M_{2}}\right]+\right. \\
& -\sin ^{2} \vartheta \sin \left(\varphi+\varphi_{S_{2}}\right) F\left[\hat{\mathbf{h}} \cdot \mathbf{p}_{1_{T}} \frac{\bar{h}_{1}^{\perp} h_{1 T}^{f}}{M_{1}}\right]+ \\
& \left.-\sin ^{2} \vartheta \sin \left(3 \varphi-\varphi_{S_{2}}\right) F\left[\left(4 \hat{\mathbf{h}} \cdot \mathbf{p}_{1_{T}}\left(\hat{\mathbf{h}} \cdot \mathbf{p}_{2_{T}}\right)^{2}-2 \hat{\mathbf{h}} \cdot \mathbf{p}_{2_{T}} \mathbf{p}_{1_{T}} \cdot \mathbf{p}_{2_{T}}-\hat{\mathbf{h}} \cdot \mathbf{p}_{1_{T}} \mathbf{p}_{2_{T}}^{2}\right) \frac{\bar{h}_{1}^{\perp f} h_{1 T}^{\perp f}}{2 M_{1} M_{2}^{2}}\right]\right\} .
\end{aligned}
$$

With respect to the unpolarised cross section, we have an additional term which depends explicitly on the Sivers function $f_{1 T}^{\perp f}$, on the Transversity $h_{1 T}^{f}$, and on the Boer-Mulders function $h_{1 T}^{\perp f}$. The Sivers function $f_{1 T}^{\perp f}$ describes the distribution of unpolarised parton inside a transversely polarised hadron, and it can be accessed through ist convolution with the unpolarised function $f_{1}^{f}$. The Transversity describes the density of transversely polarised quarks inside a transversely polarised hadron, and it is convoluted with the BM function. Those terms show a $\sin \left(\varphi-\varphi_{S_{2}}\right)$ and $\sin (\varphi+$ $\left.\varphi_{S_{2}}\right)$ azimuthal dependences to the leading twist description of the cross section. 
DY processes are investigated in the so called "safe region" in a dilepton mass range between 4 and $9 \mathrm{GeV} / \mathrm{c}^{2}$, free of resonance contributions. A second region can be defined in the range 1.5 $\leq \mathrm{M}_{\gamma^{*}} \leq 2.5 \mathrm{GeV} / \mathrm{c}^{2}$, which is free of resonance contributions. It has an higher cross section, and allows investigations on a larger kinematic region. From the simulations, an accumulation of DY events was observed at lower $\mathbf{M}_{\gamma^{*}}$ values. At the maximum beam energy available $\left(s=30 \mathrm{GeV}^{2}\right)$ the expected cross section is in the order of the nb [2]. The main background source consists of events of the type $\bar{p} p \rightarrow n\left(\pi^{+} \pi^{-}\right) X$, where $n$ indicates the number of the $\pi^{+} \pi^{-}$pairs, and its cross section is estimated to be in the order of 20-30 $\mu \mathrm{b}$ [2]. This leads to the required background rejection factor $\sim 10^{7}$.

In order to study the DY pair production in the PANDA energy range, the Drell-Yan AB event generator [7] was used. In agreement with the cross section distributions in the literature, the code generates DY muon pairs in $\bar{p}$ and $\pi^{-}$interactions with unpolarised or polarised nuclear targets.

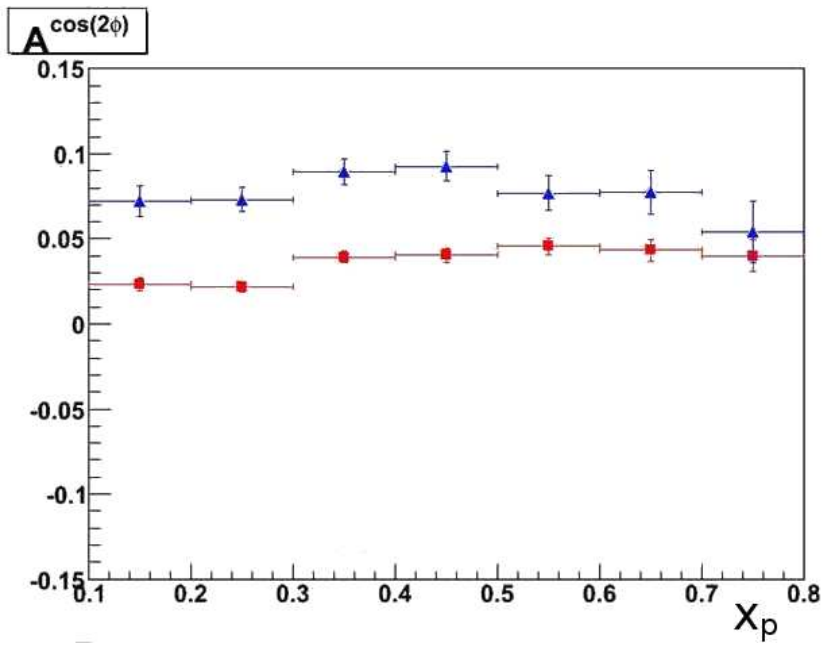

Figure 3: Simulated experimental asymmetry related to the $\cos 2 \varphi$ term, plotted as a function of $x_{p}$, longitudinal momentum fraction of the hadronic probe, for different $q_{T}$ ranges: $1 \leq q_{T} \leq 2 \mathrm{GeV} / \mathrm{c}$ (red square dots), and $2 \leq q_{T} \leq 3 \mathrm{GeV/c}$ (blue triangular dots).

In order to understand the feasibility of this measurement, 500000 events were simulated. In Fig. 3, 4, and 5 the azimuthal asymmetries are plotted as function of the longitudinal momentum of the hadronic probe $\left(x_{p}\right)$, for the unpolarized and the single-polarized case respectively; the $x$ dependence of the asymmetries included in these simulations is not relevant, and it has been introduced only in order to probe the accessible kinematic region. A selection on the different transverse momenta of the muon pair ( $\left.q_{T}\right)$ was operated: $1 \leq q_{T} \leq 2 \mathrm{GeV} / \mathrm{c}$ (square dots), and $2 \leq q_{T} \leq 3$ $\mathrm{GeV} / \mathrm{c}$ (triangular dots). Acceptance and efficiency corrections, which are crucial for such kind of measurement, are still under investigation. In the unpolarised case, the error bars are so good that a complete scan on the full transverse momenta range should be possible. Such studies should also lead to probe the balance between soft and hard processes for the DY production by investigating a possible inversion of the lepton pair transverse momentum dependency of the azimuthal asymmetries. In the single-polarised case, the scenario is more complex: the obtained distributions indicate that a transverse momentum scan could be difficult or even impossible. At the highest foreseen 


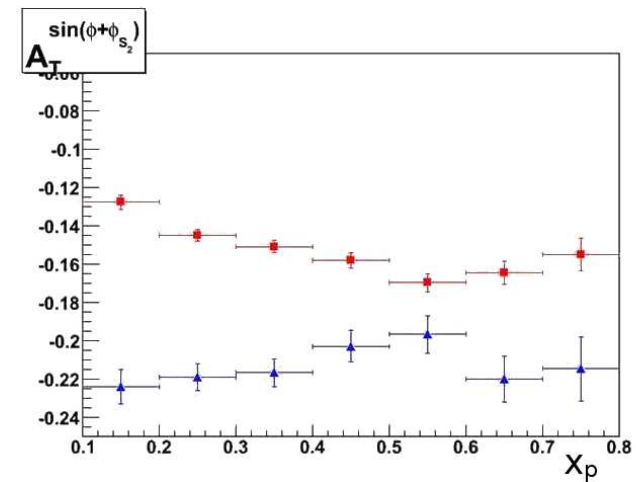

Figure 4: Simulated experimental asymmetry related to the $\sin \left(\varphi+\varphi_{S_{2}}\right)$ term, plotted as a function of $x_{p}$, longitudinal momentum fraction of the hadronic probe, for different $q_{T}$ ranges: $1 \leq q_{T} \leq 2 \mathrm{GeV} / \mathrm{c}$ (red square dots), and $2 \leq q_{T} \leq 3 \mathrm{GeV} / \mathrm{c}$ (blue triangular dots).

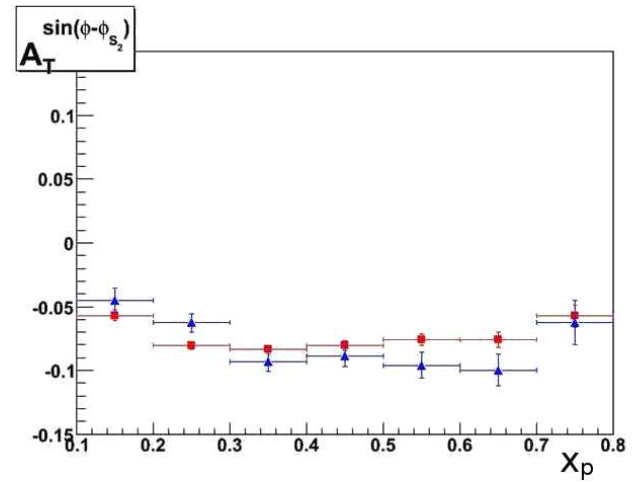

Figure 5: Simulated experimental asymmetry related to the $\sin \left(\varphi-\varphi_{S_{2}}\right)$ term, plotted as a function of $x_{p}$, longitudinal momentum fraction of the hadronic probe, for different $q_{T}$ ranges: $1 \leq q_{T} \leq 2 \mathrm{GeV} / \mathrm{c}$ (red square dots), and $2 \leq q_{T} \leq 3 \mathrm{GeV} / \mathrm{c}$ (blue triangular dots).

luminosity $\left(2 \cdot 10^{32} \mathrm{~cm}^{-2} \mathrm{~s}^{-1}\right)$, taking into account the geometrical acceptance, the material budget, and the reconstruction efficiency $(\varepsilon=0.33$ ), one year of data taking should be enough to obtain error bars similar to the simulated ones. Studies on background rejection are still going on [8].

\section{Phase between the strong and the electromagnetic $J / \psi$ decay amplitudes}

An energy scan below the $J / \psi$ peak could probe the existence of an interference between the resonant $e^{+} e^{-} \rightarrow J / \psi \rightarrow$ hadrons and non-resonant $e^{+} e^{-} \rightarrow$ hadrons amplitudes. In a model independent way, the relative phase between the strong and the electromagnetic amplitudes could be investigated by searching for interference in the $\mathrm{Q}^{2}$ behavior. In the perturbative regime, of which the relatively small $J / \psi$ decay width $(\simeq 93 \mathrm{KeV})$ could be interpreted as a proof, the $J / \psi$ resonant amplitudes, the strong $A_{3 g}$ (Fig. 6a) and the electromagnetic $A_{\gamma}$ (Fig. 6b) ones, are expected to be almost real [9]. The non resonant electromagnetic amplitude $A_{E M}$ (Fig. 6c) is expected to be real as well. If those amplitudes are all real, they are expected to interfere, with a relative phase $\sim 0^{\circ}$. On the contrary, the experimental data $[9,10]$ indicate an unexpected phase difference of $\sim 90^{\circ}$ in the $J / \psi$ decays into $N \bar{N}, V P, P P$, and $V V$ (where $\mathrm{N}, \mathrm{V}$ and $\mathrm{P}$ stand for nucleon, vector and pseudoscalar meson respectively), which correspond to no interference pattern. Such an experimental scenario points hence toward relevant imaginary amplitudes. At the moment, QCD does not provide an explanation of such phase difference, or so large imaginary amplitudes [11].

In the inclusive $J / \psi$ decay, the interference term should vanish, since one is summing up on all the decay channels, as observed experimentally. However, it should be possible to observe this interference term in all the exclusive channels. The PANDA experiment will have the unique possibility to investigate reactions like $\bar{p} p \rightarrow J / \psi \rightarrow \mu^{+} \mu^{-}$. With respect to the existing $e^{+} e^{-}$ collider experiment as BESIII, which will measure the phase in the exclusive channels $e^{+} e^{-} \rightarrow$ $J / \psi \rightarrow N \bar{N}, V P, P P, V V$ in the next future, the radiative corrections in the initial state are not needed 


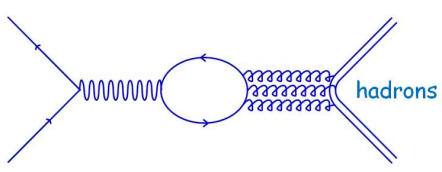

(a)

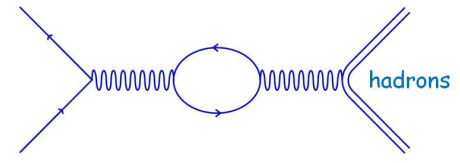

(b)

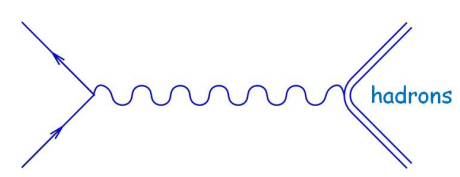

(c)

Figure 6: Diagram for the process $e^{+} e^{-} \rightarrow$ hadrons: (a) strong $A_{3 g}$, (b) electromagnetic $A_{\gamma}$, and (c) non resonant $A_{E M}$ contributions.

when considering $\bar{p} p$ interactions. In Fig. 7, the simulated cross sections respectively for a $0^{\circ}$ (black), $90^{\circ}$ (red), and $180^{\circ}$ (blue) phase for the process $e^{+} e^{-} \rightarrow p \bar{p}$ are shown. The effect of the radiative corrections reflects in a huge spreading of the deep at $0^{\circ}$ phase. On the contrary, the simulated yelds for $\bar{p} p \rightarrow \mu^{+} \mu^{-}$, shown in Fig. 8, indicate a narrow deep in corrispondence of the $0^{\circ}$ phase cross section. With the PANDA spectrometer it could be possible to observe largely different cross sections, by almost 4 order of magnitude, at the maximum interference ( $0^{\circ}$ phase).

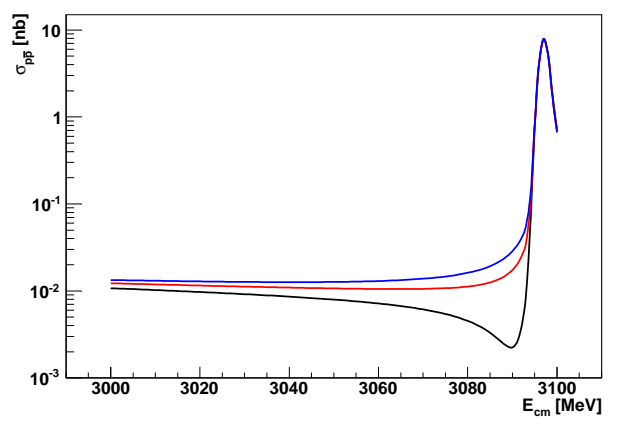

Figure 7: Expected interference pattern for the process $e^{+} e^{-} \rightarrow p \bar{p}$, for different phases between the $J / \psi$ decay amplitudes: $0^{\circ}$ (black line), $90^{\circ}$ (red line), and $180^{\circ}$ (blue line).

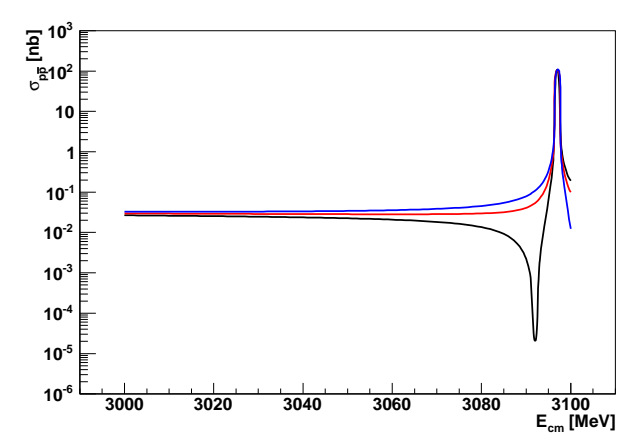

Figure 8: Expected interference pattern for the process $\bar{p} p \rightarrow \mu^{+} \mu^{-}$, for different phases between the $J / \psi$ decay amplitudes: $0^{\circ}$ (black line), $90^{\circ}$ (red line), and $180^{\circ}$ (blue line).

The choice of the energy points is crucial for this measurement. In the case of maximum interference, a reasonable choice would be to take at least 5 points. The first 2 points should be far from the deep position, in order to fix the continuum value (the cross section value far from the $J / \psi$ resonance) and the cross section slope. Other 2 points could be taken around the deep position, and the last one at the beginning of the $J / \psi$ Breit-Wigner. The experimental data available in the literature point toward a $90^{\circ}$ phase. In this scenario, one could investigate the gradient of the cross section in order to define the mass region where the sensibility is higher. Keeping apart the first two points, it was observed that the deep corresponds roughly to the maximum gradient region.

Inverstigations on the phase precision are ongoing. Optimization of the phase reconstruction and of the full procedure is now in progress.

\section{Acknowledgements}

This work was supported in part by Università degli Studi di Torino, Regione Piemonte, and INFN Torino. 


\section{References}

[1] M. Kotulla, et al., PANDA Technical Progress Report, Strong Interaction Studies with Antiproton, (2005)

[2] W. Erni, et al., Physics Performance Report for: PANDA, Strong Interaction Studies with Antiproton, (2009) arXiv:0903.3905

[3] W. Erni, et al., Technical Design Report for the PANDA Muon System, $2^{n} d$ Draft (May 2011)

[4] E.Iarocci, Plastic streamer tubes and their applications in high energy physics, Nucl. Instr. and Meth. 217, 30 (1983)

[5] C. Bourrely and J. Soffer, Do we understand the single-spin asymmetry for $\pi^{0}$ inclusive production in pp collisions?, Eur. Phys. J. C36, 371 (2004)

[6] J. C. Collins and D. E. Soper, Angular distribution of dileptons in high-energy hadron collisions, Phys. Rev. D16, 2219 (1977)

[7] A. Bianconi, A scheme for fast exploratory simulation of azimuthal asymmetries in Drell-Yan experiments at intermediate energies. The DY_AB Monte Carlo event generator, Nucl. Inst. Meth. A593:562-571 (2008)

[8] M. Destefanis, Spin Physics at FAIR, PoS (Bormio 2011) 012, (2011)

[9] R. Baldini, C. Bini, E. Luppi, Phys. Lett. B404, 362 (1997)

[10] R. Baldini et al., Phys. Lett. B444, 111 (1998); J.M. Bian, $J / \psi->$ ppbar and $J / \psi->$ nnbar measurement by BESIII, approved draft; L. Kopke and N. Wermes, Phys. Rep. 174, 67 (1989); J. Jousset et al., Phys. Rev. D41,1389 (1990); M. Suzuki et al., Phys. Rev. D60, 051501 (1999)

[11] J. Bolz and P. Kroll, WU B 95-35 